\title{
Molecular characterization and phylogenetic analysis of the complete genome of a porcine sapovirus from Chinese swine Shixing Yang ${ }^{\dagger 1}$, Wen Zhang ${ }^{\dagger 2}$, Quan Shen ${ }^{1}$, Fen Huang ${ }^{1}$, Yan Wang ${ }^{1}$, Jianguo Zhu' ${ }^{1}$, Li Cui1 ${ }^{1}$, Zhibiao Yang1 and Xiuguo Hua*1
}

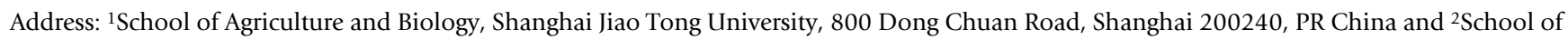
Medical Science and Laboratory Medicine, Jiangsu University, 301 Xuefu Road, Zhenjiang, Jiangsu 212013, PR China

Email: Shixing Yang - yangshixing@sjtu.edu.cn; Wen Zhang - z0216wen@yahoo.com; Quan Shen - shenquanfly@yahoo.com; Fen Huang - huangfen6789@163.com; Yan Wang - catcatmy@sjtu.edu.cn; Jianguo Zhu - jgzhu@sjtu.edu.cn; Li Cui - lcui@sjtu.edu.cn; Zhibiao Yang - zbyang@sjtu.edu.cn; Xiuguo Hua* - hxg@sjtu.edu.cn

* Corresponding author †Equal contributors

Published: 6 December 2009

Virology Journal 2009, 6:216 doi:10.1186/1743-422X-6-216

This article is available from: http://www.virologyj.com/content/6/1/216

(C) 2009 Yang et al; licensee BioMed Central Ltd.

This is an Open Access article distributed under the terms of the Creative Commons Attribution License (http://creativecommons.org/licenses/by/2.0), which permits unrestricted use, distribution, and reproduction in any medium, provided the original work is properly cited.

\begin{abstract}
Background: Porcine sapovirus was first identified in the United States in 1980, hitherto, several Asian countries have detected this virus. In 2008, the first outbreak of gastroenteritis in piglets caused by porcine sapovirus in China was reported. The complete genome of the identified SaV strain Ch-sw-savl was sequenced and analyzed to provide gene profile for this outbreak.
\end{abstract}

Methods: The whole genome of Ch-sw-savl was amplified by RT-PCR and was sequenced. Sequence alignment of the complete genome or RNA dependent RNA polymerase (RdRp) gene was done. $3^{\prime}$ end of ORF2 with $2 \mathrm{I}$-nt nucleotide insertion was further analyzed using software.

Results: Sequence analysis indicated that the genome of Ch-sw-savl was 754I nucleotide long with two ORFs, excluding the 17 nucleotides ploy $(A)$ at the $3^{\prime}$ end. Phylogenetic analysis based on part of RdRp gene of this strain showed that it was classified into subgroup GIII. Sequence alignment indicated that there was an inserted $2 \mathrm{I}$-nt long nucleotide sequence at the $3^{\prime}$ end of ORF2. The insertion showed high antigenicity index comparing to other regions in ORF2.

Conclusion: Ch-sw-savl shared similar genetic profile with an American PEC strain except the $2 \mathrm{I}$-nt nucleotide at the $3^{\prime}$ end of ORF2. The insert sequence shared high identity with part gene of Sus scrofa clone RP44-484MIO.

\section{Background}

Caliciviridae is a family of positive sense single-stranded RNA viruses comprised of both human and animal pathogens [1]. Caliciviridae family contains four genera, Lagovirus, Vesivirus, Norovirus and Sapovirus [2]. Various caliciviruses possess common features. For example, they are small, non-enveloped virus, $27-38 \mathrm{~nm}$ in diameter. They possess a single-stranded, 7.3-8.3 kb plus-sense RNA genome, a single 56-71 kD capsid protein [3], and a polyprotein containing confering motifs of a putative $2 \mathrm{C}$ helicase, 3C-like protease, and 3D RdRp. SaV are recognized as emerging enteric pathogens in humans, swine and mink [4]. SaV infection may cause diarrhea especially in the younger [5]. It is currently divided into eight distinct genetic groups (GI-GVIII) based on the RdRp gene. Among these genetic groups, GIII can't infect humans but 
can be cultured in vitro in the presence of bile acid [6]. The genome of $\mathrm{SaV}$ consists of 7.1-7.5 kb nucleotide and encodes two or three open reading frames (ORFs). ORF1 encodes one polyprotein that contains coding sequences for the nonstructural proteins and the major capsid protein (VP1), ORF2 encodes the minor structural protein (VP2), while ORF3 is only present in strains from genotypes GI, GIV and GV, and encodes a small basic protein [7]. SaV is considered as a significant global enteropathogen of acute gastroenteritis [8]. Recently, it was shown that the host tropism of some calicivirus is less specific. Some calicivirus may have zoonotic potential, and animals such as domestic pig may be a reservoir for caliciviruses [9-11]. Porcine sapovirus was first identified in the United States by electron microscopy in 1980 [12] and genetically characterized as a sapovirus in 1999 [13]. Recently, SaV infections have been identified in Japan, South Korea, Venezuela, Hungary and Belgium [14-18]. In the United States, porcine sapovirus was also detected from Oyster [19]. Although porcine SaV was mainly detected in pigs, some studies indicated that some porcine $\mathrm{SaV}$ might be potential pathogencity transmitting to humans. For example, the porcine SaV strain (Sapovirus pig/43/06-18p3/ 06/ITA) isolated from Italy was most closely related to human SaV through the alignment of RdRp sequences, suggesting the possibility of a pig reservoir for human strains or vice versa [20]. We previously reported an outbreak of gastroenteritis in piglets in China caused by the first Chinese porcine SaV strain [21]. In this study, gene profile of this strain was investigated, the entire viral genome and 3' end of Ch-sw-sav1 were cloned and sequenced.

\section{Methods \\ Samples}

Porcine SaV positive fecal samples were collected from commercial pig farms in Shanghai as introduced in our previous study. Samples were converted to 20\% (wt/vol) suspensions in phosphate-buffered saline (PBS) $(0.01 \mathrm{M}$, $\mathrm{pH} 7.2$ to 7.4 ) and clarified by centrifugation at $10,000 \mathrm{~g}$ for $10 \mathrm{~min}$.

\section{Primers Design}

In order to amplify the full-length sequence, 15 sets of primers were designed based on the sequences of $\underline{\text { AF18276 }}$ and DQ056363 that were previously submitted in the GenBank: Nucleotide sequence and position of the primers are listed in Table 1.

Table I: Nucleotide sequences of the oligonucleotides used for PCR amplification and sequencing

\begin{tabular}{|c|c|c|c|}
\hline Primer set & Primer name & Nucleotide sequence & Position \\
\hline \multirow[t]{2}{*}{ I } & SPIF & GTGATCGGTGATGGCTAATTGCCG & $1-14$ \\
\hline & SPIR & TGGAGATGGTATCTGTCAGTGTG & $645-667$ \\
\hline \multirow[t]{2}{*}{2} & $\mathrm{SP} 2 \mathrm{~F}$ & GGCAGTACATTTGTGAGGGGTG & $543-564$ \\
\hline & SP2R & ССTGTTCTGCTTTATCACСTCC & $1170-1191$ \\
\hline \multirow[t]{2}{*}{3} & SP3F & GACGGTGGCTGCCATTAAAGCTG & $1063-1085$ \\
\hline & SP3R & GCAGTGTAGCCGCGTACTGAGC & $1833-1854$ \\
\hline \multirow[t]{2}{*}{4} & SP4F & ATTGACGTGACAGCCCCCAC & $1733-1752$ \\
\hline & SP4R & TGTGGTTCTTGACTGGTGAG & $2335-2354$ \\
\hline \multirow[t]{2}{*}{5} & SP5F & TGGTGGAGGCCTGTTCAGAGC & $2223-2243$ \\
\hline & SP5R & CCAAGTTGTGGGCTGTCAACAC & $2757-2778$ \\
\hline \multirow[t]{2}{*}{6} & SP6F & CAGAGTCCTCCTGGTGGACATTC & $2680-2702$ \\
\hline & SP6R & ATTACCAAGCGCAACGCTAGGC & $3340-3361$ \\
\hline \multirow[t]{2}{*}{7} & SP7F & CATGTGGCCAACATGTGTG & $3243-3261$ \\
\hline & SP7R & TGATTTGGTCAAGGTAGCC & $3873-3891$ \\
\hline \multirow[t]{2}{*}{8} & SP8F & ССТTСTACAACACCAAATGATTGCC & $3768-3792$ \\
\hline & SP8R & AGGCCAGGATGTCAACACTGGCAC & $437 \mid-4394$ \\
\hline \multirow[t]{2}{*}{9} & SP9F & ATGTATGGATAGCCCTCAGATTG & $4261-4283$ \\
\hline & SP9R & GTCCACATCAACGGCCGCCGGCTCG & $4890-4914$ \\
\hline \multirow[t]{2}{*}{10} & SPIOF & AGCCAACAGACACTCCTGTGTTCC & $4760-4783$ \\
\hline & SPIOR & CATGCCAGACCCTGATATTATCACC & $5468-5492$ \\
\hline \multirow[t]{2}{*}{ II } & SPIIF & ACCTACACCAATGTCACCTGGAC & $5328-5350$ \\
\hline & SPIIR & GTGCCACACCTACTATGACCACAG & $5890-5913$ \\
\hline \multirow[t]{2}{*}{12} & $\mathrm{SPI} 2 \mathrm{~F}$ & TCAAGCCTCCAAACCAAGCC & $5784-5803$ \\
\hline & SPI2R & TGGCGGTCCATAAATGAGGTG & $6395-6415$ \\
\hline \multirow[t]{2}{*}{13} & SPI3F & TATGCAGCTTTGGCAATTCCC & $6291-6311$ \\
\hline & SPI3R & TTGATCTTTAGCAACTGTATCTG & $6892-6915$ \\
\hline \multirow[t]{2}{*}{14} & SPI4F & TTGGATTGCAGGAGCAATGCAGG & $6777-6799$ \\
\hline & SPI4R & TGTAAGGTTCGGTACGCGTAACC & $7280-7303$ \\
\hline \multirow[t]{2}{*}{15} & SPI5FI & TCAATTGGCTGGGTCACGTGAAG & $7027-7049$ \\
\hline & SPI5F2 & CAAACACCTTTGGTCCACCAAGG & $7070-7092$ \\
\hline
\end{tabular}


Table 2: Summary of sapovirus strains and representative strains for Lagovirus, Vesivirus, and Norovirus genera and NB-like viruses used in sequence analysis

\begin{tabular}{|c|c|c|}
\hline Strains & Genus/genogroup & GenBank accession no. \\
\hline Sapovirus Mcl0/Japan & $\mathrm{SaV} / \mathrm{GII}$ & NC 010624 \\
\hline Sapovirus C12/Japan & $\mathrm{SaV} / \mathrm{GII}$ & $\overline{A Y 603425}$ \\
\hline Sapovirus SaKaeo-15/Thailand & $\mathrm{SaV} / \mathrm{GII}$ & AY646855 \\
\hline Sapovirus Mc2/Japan & $\mathrm{SaV} / \mathrm{GII}$ & AY237419 \\
\hline Sapovirus Ehime I I07/2002/JP & $\mathrm{SaV} / \mathrm{GII}$ & $\overline{D Q 058829}$ \\
\hline Sapovirus Mcl I4/Japan & $\mathrm{SaV} / \mathrm{GI}$ & $\overline{A Y 237422}$ \\
\hline Sapovirus Hu/Dresden/pJG-Sap0I/DE & $\mathrm{SaV} / \mathrm{Gl}$ & AY694184 \\
\hline Sapovirus NongKhai-24/Thailand & $\mathrm{SaV} / \mathrm{GV}$ & AY646856 \\
\hline Porcine enteric sapovirus/USA & SaV/GIII & $\mathrm{AFI} 82760$ \\
\hline Norovirus mouse/Hannoverl/2007/DEU & Mouse NoV & EU854589 \\
\hline Norwalk virus/USA & NoV/GI & $\mathrm{NC001959}$ \\
\hline Norwalk virus/Germany & $\mathrm{NoV} / \mathrm{GI}$ & AF093797 \\
\hline Norovirus Hu/GI/Otofuke// 979/JP & $\mathrm{NoV} / \mathrm{GI}$ & $\overline{\mathrm{AB}|875| 4}$ \\
\hline Bovine calicivirus/UK & Bovine calicivirus & A 011099 \\
\hline Bo/Dumfries/94/UK & Bovine calicivirus & AY 126474 \\
\hline Human calicivirus strain Mc37/Japan & NoV/GII & $\overline{\mathrm{AY} 237415}$ \\
\hline Norwalk-like virus/Gifu'96/Japan & NoV/GII & $\overline{\mathrm{AB} 045603}$ \\
\hline Hawaii calicivirus/USA & NoV/GII & HCU076II \\
\hline Lordsdale virus & NoV/GII & $\underline{X 86557}$ \\
\hline Norovirus Hu/GII-4/Hokkaidol/2006/JP & NoV/GII & $\mathrm{AB} 447427$ \\
\hline Norovirus Hu/Houston/TCHI86/2002/US & NoV/GII & EU310927 \\
\hline Norovirus Hu/NLV/Oxford/B4S4/2002/UK & NoV/GII & AY587986 \\
\hline Feline calicivirus & $\mathrm{FCV}$ & M86379 \\
\hline San Miguel sea lion virus serotype I & $\underline{\text { SMSVI }}$ & SMUI530I \\
\hline European brown hare syndrome virus & $\overline{\mathrm{RHDV}}$ & $M 67473$ \\
\hline European brown hare syndrome virus & EBHSV & $\underline{Z 69620}$ \\
\hline
\end{tabular}

\section{RNA extraction and cDNA synthesis}

Viral RNA was extracted with TRIzol Reagent from supernatants of fecal suspensions, according to the manufacture's instructions. The cDNA synthesis was primed by Oligo $\mathrm{dT}_{16}$ or the reverse one of each set of primers using TaKaRa RNA PCR kit (TaKaRa, Japan) in a $10 \mu \mathrm{L}$ reaction volume. The reaction condition was $40 \mathrm{~min}$ at $42^{\circ} \mathrm{C}$, then $15 \mathrm{sec}$ at $86^{\circ} \mathrm{C}$.

\section{PCR and RACE amplifications of the full-length SaV genome}

PCR was carried out in $50 \mu \mathrm{L}$ reaction volume, containing $8 \mu \mathrm{L}$ dNTP Mixture ( $25 \mathrm{mM}$ ), $5 \mu \mathrm{L} 10 \times$ Ex-taq buffer, 0.2 $\mu \mathrm{L}$ Ex Taq, $1 \mu \mathrm{L}(25 \mathrm{mM})$ of each primer, $10 \mu \mathrm{L}$ of template and adding sterilize $\mathrm{H}_{2} \mathrm{O}$ to $50 \mu \mathrm{L}$. The reaction was done with the following profile: Activation of DNA polymerase at $95^{\circ} \mathrm{C}$ for $5 \mathrm{~min}$, followed by 35 cycles of denaturation of DNA at $95^{\circ} \mathrm{C}$ for $40 \mathrm{sec}$, annealing at the $50^{\circ} \mathrm{C}$ for $1 \mathrm{~min}$, extension at $72^{\circ} \mathrm{C}$ for $1 \mathrm{~min}$ and then followed by a final extension step at $72^{\circ} \mathrm{C}$ for $10 \mathrm{~min}$. Purfied PCR products were ligated to pMD-18T vector (TaKaRa, Japan) and 3 to 5 positive colonies were sequenced.

\section{3' RACE}

The 3' RACE was carried out with TaKaRa RNA PCR Kit (TaKaRa, Japan) following the manufacture's instructions.
Briefly, ten microliters of RNA were used as template to synthesize cDNA with AMV Reverse transcriptase for $1 \mathrm{~h}$ at $42^{\circ} \mathrm{C}$. The external reverse primer which has a poly $(\mathrm{T})$ tract was used to prime the cDNA synthesis. The cDNA was then amplified with the external forward primer (5'TCAATTGGCTGGG TCACGTGAAG-3', nucleotide position numbers 7027-7049) and internal forward primer (5'- CAAACACCTTTGGTCCACCAAGG-3', nucleotide position numbers 7070-7092) with Ex Taq DNA polymerase (TaKaRa, Japan). The PCR reaction mixture was incubated for $2 \mathrm{~min}$ at $94^{\circ} \mathrm{C}$, followed by 35 amplification cycles comprising denaturation at $94^{\circ} \mathrm{C}$ for $30 \mathrm{~s}$, annealing at $65^{\circ} \mathrm{C}$ for $30 \mathrm{~s}$, and extension at $72^{\circ} \mathrm{C}$ for $30 \mathrm{~s}$. The product was extended for another $7 \mathrm{~min}$ at $72^{\circ} \mathrm{C}$ to ensure a full extension.

The PCR products were purified from 1\% agarose gel using the QIAquick Gel Extraction kit (Qiagen, Gemany). Purified PCR products were ligated into pMD18-T Vector. For each product, three to five positive colonies were selected and sequenced.

\section{Phylogenetic analysis}

Nucleotide sequences of the following calicivirus in Genbank were used in the phylogenic analysis (Table 2): SVs: Sapovirus Mc10/Japan (NC 010624), Sapovirus C12/ Japan (AY603425), Sapovirus SaKaeo-15/Thailand 

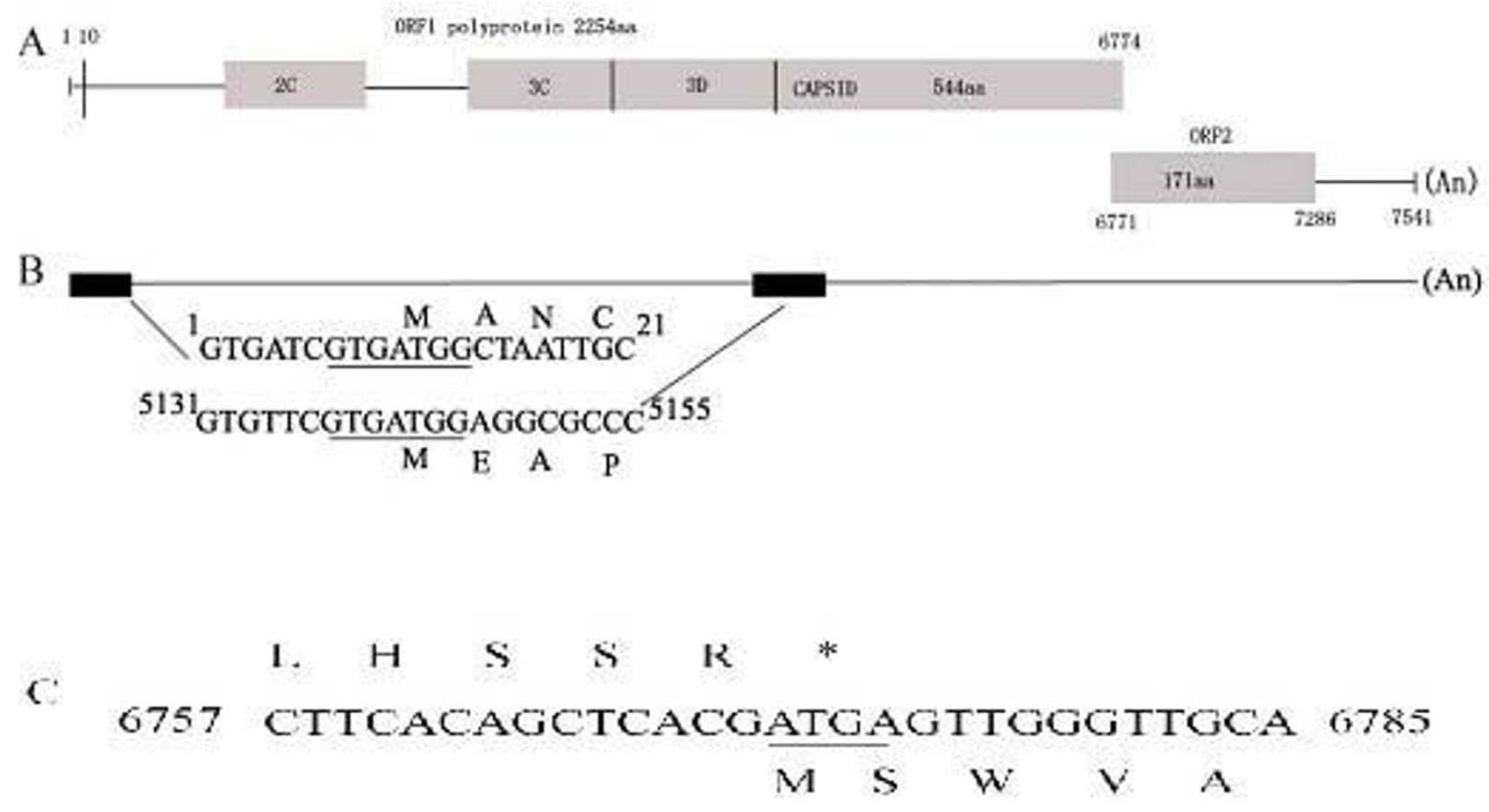

Figure I

Genomic characteristic of Ch-sw-sav I. A. Schematic of the genomic organization of Ch-sw-savl showing the two predicted ORFs: ORFI, encoding a polyprotein fused to and contiguous with the capsid protein (VPI), forming a large polyprotein; and ORF2 encoding a small basic protein (VP2) of unknown function. B. Schematic of the conserved nucleotide sequence motifs at the $5^{\prime}$ termini of the genomic and predicted subgenomic RNAs. The Kozak context, favorable for translation initiation, is underlined. C. Aligned nucleotide and predicted amino acid sequences at the junction between ORFI and ORF2. ORF2 overlaps the 3 ' end of ORFI by 4 nt (underlined).

(AY646855), Sapovirus Mc2/Japan (AY237419), Sapovirus Ehime1107/2002/JP(DQ058829), Sapovirus Mc114/ Japan (AY237422), Sapovirus Hu/Dresden/pJG-Sap01/ DE (AY694184), Sapovirus NongKhai-24/Thailand (AY646856), and Porcine enteric sapovirus/USA ( $\underline{\text { AF182760) }}$; NVs: Norovirus mouse/Hannover1/2007/ DEU (EU854589), Norwalk virus/USA (NC001959), Norwalk virus/Germany (타093797), Norovirus Hu/GI/ Otofuke/1979/JP (AB187514), Bovine calicivirus/UK

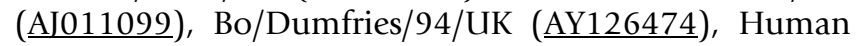
calicivirus strain Mc37/Japan (AY237415), Norwalk-like virus/Gifu'96/Japan (AB045603), Hawaii calicivirus/USA (HCU07611), Lordsdale virus ( $\underline{\text { X6557) }}$, Norovirus Hu/ GII-4/Hokkaido1/2006/JP (셔447427), Norovirus Hu/ Houston/TCH186/2002/US (EU310927), Norovirus Hu/ NLV/Oxford/B4S4/2002/UK (AY587986); VVs: FCV (M86379) and SMSV1 (SMU15301); LVs: RHDV (M67473) and EBHSV (Z69620). Sequencing reads from each PCR product were assembled using SeqMan II program (DNASTAR, Inc). Multiple sequence alignment was performed using CLUSTAL $\mathrm{W}$ method. The nucleotide identity and nucleotide divergence between complete Por- cine $\mathrm{SaV}$ genomes was calculated using MegAlign program (DNASTAR, Inc). MEGA software was used to construct a phylogenetic tree, the reliability of the generated tree was evaluated by bootstrapping 1000 replicates. The same process was applied to analyse part of RNA dependent RNA polymerase genes, Nucleotide sequences of the following calicivirus in Genbank were used in the phylogenic analysis: Sapovirus $\mathrm{Hu} / \mathrm{Lyon} / 30338 / 98 / \mathrm{F}$ (AJ251991), Sapporo virus-Manchester ( $\underline{\text { X6560) }})$, Sapporo virus-Houston/86 (ㄴ5643), Sapovirus Hu/Ehime/ 2K-814/2000 (AJ606698), Sapovirus Hu/Potsdam/2000/ DEU (AF294739), Sapovirus Hu/Mex14917/2000 (AF435813), Sapovirus Hu/Hou7-1181 (AF435814), Sapovirus Hu/Ehime/99-1596/1999/JP (AJ606697), Sapovirus Hu/Ehime/01-1669/2001 (시66699), Sapovirus $\mathrm{Hu} / \operatorname{Arg} 39 / 1995 / \mathrm{ARG}$ (AY289803), Sapovirus pig/43/ 06-18p3/06/ITA (EU221477), Sapovirus Hu/Chiba/ 991172/1999 (AJ606691), Sapovirus Hu/cruise ship/ 2000/USA (AY289804), Sapovirus Hu/Bristol/1998/UK (AJ249939), Sapporo virus-London/29845 (U95645), $\mathrm{Po} / \mathrm{SaV} / \mathrm{Giessen}-08 / 2003 / \mathrm{DE}$ (EU122248), Po/SaV/Giessen-07/2004/DE (EU122246), Porcine enteric sapovirus 
Table 3: Percentages of nucleotide sequence identity of Ch-sw-sav I with other caliciviruses in regions aligned for phylogeny

\begin{tabular}{|c|c|c|c|}
\hline Strain & Genogroupe & GenBank accession no. & \% Identity \\
\hline Hu/Lyon/30338/98/F & Gl & A) 251991 & 47.7 \\
\hline Sapporo virus-Manchester & GI & $\underline{X 86560}$ & 42.7 \\
\hline Sapporo virus-Houston/86 & GI & $\underline{\text { U95643 }}$ & 48.5 \\
\hline Hu/Ehime/2K-8I4/2000 & $\mathrm{Gl}$ & A] 606698 & 40.3 \\
\hline Hu/Potsdam/2000/DEU & GI & AF294739 & 57.1 \\
\hline Hu/MexI49I7/2000 & Gl & AF435813 & 40.4 \\
\hline $\mathrm{Hu} / \mathrm{Hou} 7-\mathrm{II} \mid \mathrm{I}$ & GIV & $\overline{\mathrm{AF} 435814}$ & 50.3 \\
\hline Hu/Ehime/99-1596//999/JP & GIV & A] 606697 & 45.5 \\
\hline Hu/Ehime/0I-I669/200I & GV & A]606699 & 43.8 \\
\hline Hu/Arg39/1995/ARG & GV & AY289803 & 42.1 \\
\hline pig/43/06-18p3/06/ITA & GVIII? & EU221477 & 29.0 \\
\hline $\mathrm{Hu} / \mathrm{Chiba} / 991$ I 72/1999 & GII & A)606691 & 40.0 \\
\hline Hu/cruise ship/2000/USA & GII & AY289804 & 22.9 \\
\hline Hu/Bristol/I998/UK & GII & A]249939 & 43.2 \\
\hline Sapporo virus-London/29845 & GII & $\underline{\text { U95645 }}$ & 47.2 \\
\hline Po/SaV/Giessen-08/2003/DE & GIII & EUI22248 & 88.4 \\
\hline Po/SaV/Giessen-07/2004/DE & GIII & EUI22246 & 86.1 \\
\hline swine/YiYI/2006/PRC & GIII & EU38|231 & 84.2 \\
\hline Porcine sapovirus/Venezuelan & GIII & $\underline{D Q 056363}$ & 86.1 \\
\hline swine/OH-JJ259/00/US & GIII & $\overline{\text { AY826423 }}$ & 86.1 \\
\hline Porcine enteric sapovirus/Japan & GIII & AB242875 & 84.5 \\
\hline swine/OH-MM280/03/US & GIII & AY823308 & 82.2 \\
\hline swine/NC-QW270/03/US & GIII & AY826426 & 86.7 \\
\hline PEC/swine-Id3/2005/HUN & GIII & DQ383274 & 91.2 \\
\hline Porcine enteric sapovirus/K8/JP & GVI & $\underline{A B 242873}$ & 20.2 \\
\hline Po/2053P4/Brazil & GVI & DQ359100 & 18.6 \\
\hline $\mathrm{Po} / \mathrm{OH}-\mathrm{J} / 68 \mathrm{I} / 2000 / \mathrm{US}$ & GVI & AY974192 & 28.3 \\
\hline Po/20I4P2/Brazil & GVI & DQ359099 & 16.3 \\
\hline $\mathrm{Po} / \mathrm{OH}-\mathrm{LL} 26 / 2002 / \mathrm{US}$ & GVII & AY974195 & 29.2 \\
\hline Porcine enteric sapovirus/K7/JP & GVII & AB221130 & 18.4 \\
\hline
\end{tabular}

swine/YiY1/2006/PRC (EU381231), Porcine enteric sapovirus/Venezuelan (DQ056363), Sapovirus swine/OHJJ259/00/US (AY826423), Porcine enteric sapovirus/ Japan ( $(\underline{A Y 823308}), \quad$ Sapovirus swine/NC-QW270/03/US ( $\underline{\text { AY826426) }}$, PEC/swine-Id3/2005/HUN (DQ383274), Porcine enteric sapovirus/K8/JP (AB242873), Sapovirus Po/2053P4/Brazil (DQ359100), Sapovirus Po/OH-JJ681/ 2000/US (AY974192), Sapovirus Po/2014P2/Brazil (DQ359099), Sapovirus Po/OH-LL26/2002/US (AY974195), Porcine enteric sapovirus/K7/JP (AB221130). The sequence determined in current study was deposited in GenBank, the name was Ch-sw-sav1 and the accession number was FJ387164.

\section{3' end of ORF2 partial sequences analysis}

Six available Porcine SaVs partial sequences of 3' end of ORF2 were retrieved from GenBank, according to sequence alignment. As follows: OH-MM-280-03-US

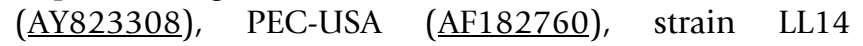
(AY425671), OH-JJ-259-00-US (AY826423), NC-QW270-03-US (AY826426). Nucleotide sequence and pro- tein were aligned by CLUSTAL W method using DNAstar software, antigen index was analysed by protean using DNAstar software.

\section{Results \\ Genomic organization of Ch-sw-savl virus}

The complete RNA genome of Ch-sw-sav1 is consisted of $7541 \mathrm{nt}$, excluding its 3' end poly(A) tail, was longer than the USA strain (GenBank no.: AF182760). It's A, C, G, U ribonucleotide composition was $19 \%, 14.3 \%, 33.3 \%$, and $33.3 \%$, respectively. The 5 ' terminus genomic RNA started with the featured trinucleotide GTG. Similar to the genomes of SVs and LVs, the Ch-sw-sav1 genome contained two predicted ORFs. ORF1 was 6765 bases $(2255$ aa) in length encoding non-structural proteins and VP1 (544aa). ORF2, consisting of 516 bases (nt 6771-7286), was predicted to encode VP2 protein with 172 aa. (Fig. 1A). The predicted polyprotein encoded by ORF1 contained the common 2C helicase (GPPGIGKT), 3C protease (GDCG), and RdRp (GLPSG and YGDD) motifs that were highly conserved in all calicivirus. The PPG motif was also present in the predicted VP1 (data not shown). 


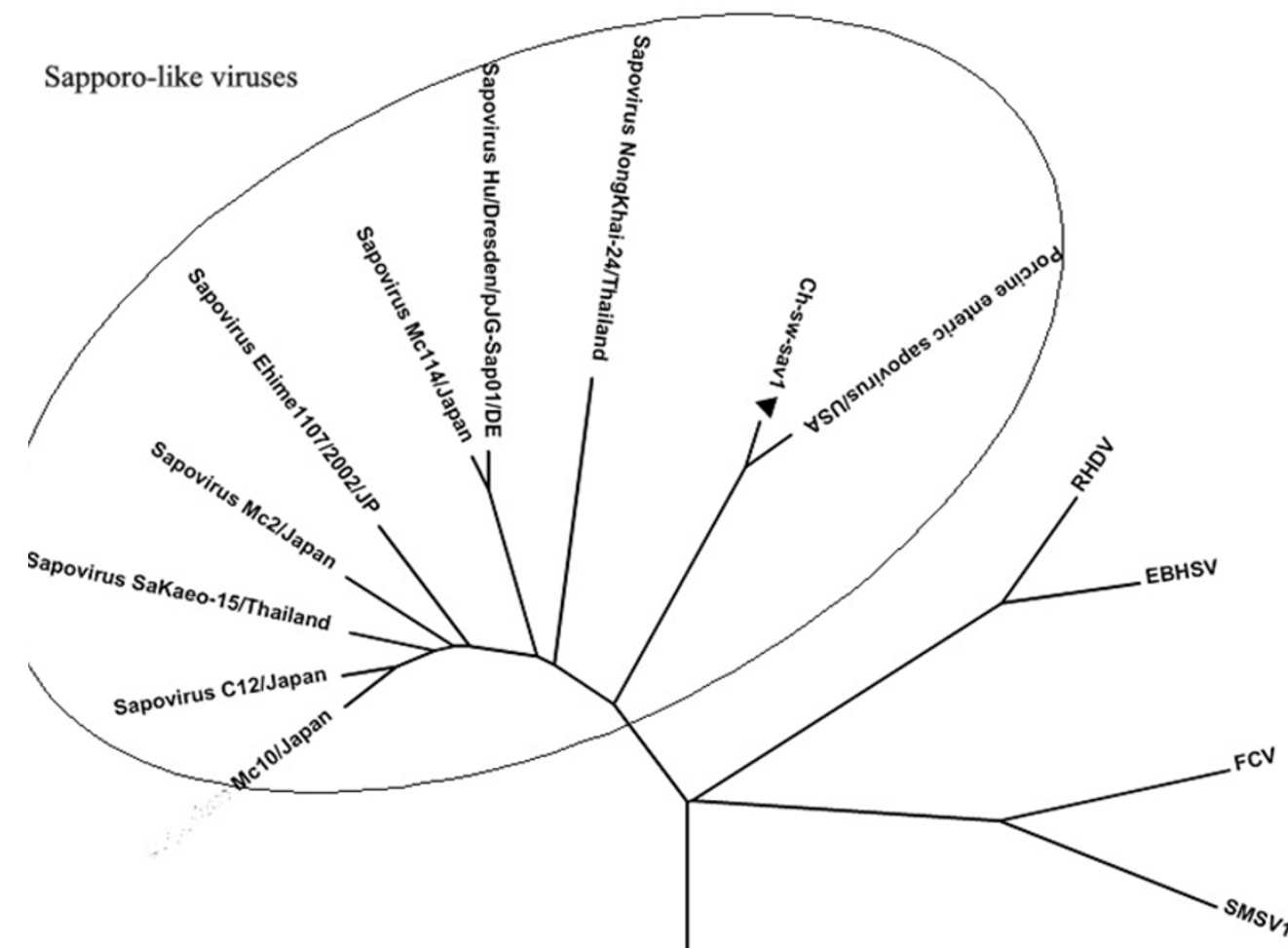

Norwalk-like viruses

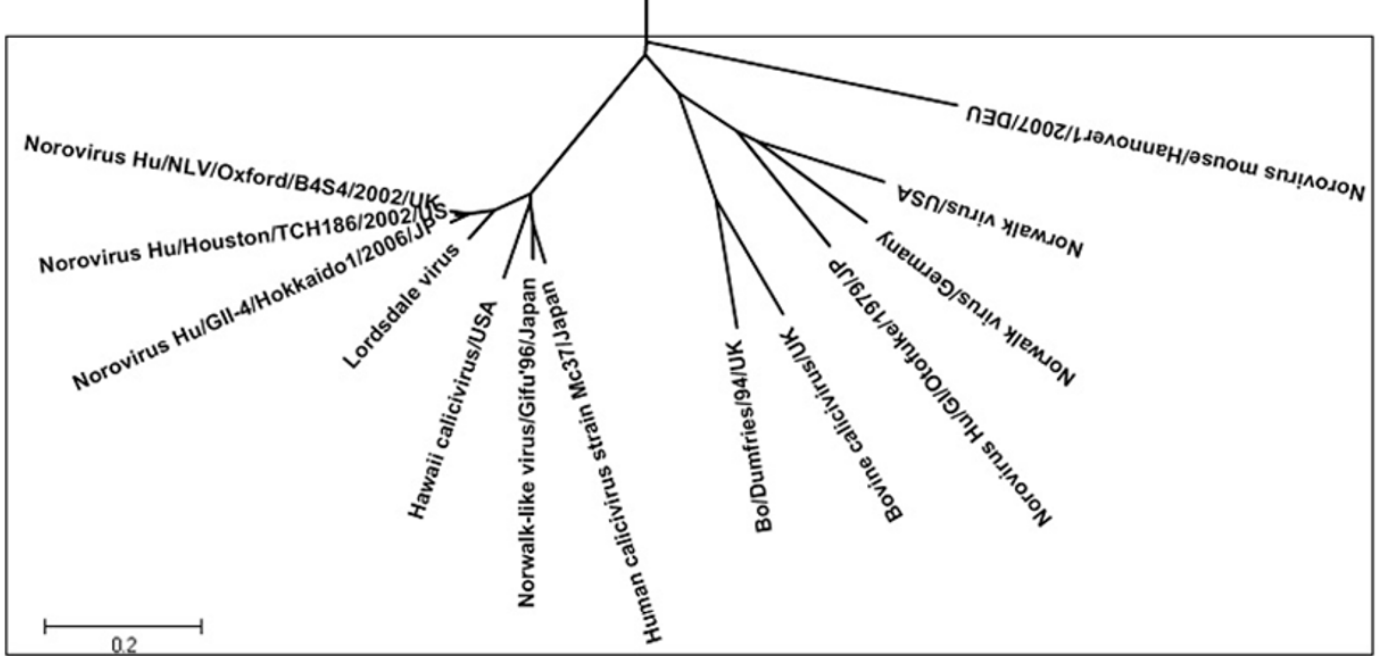

Figure 2

Phylogenetic tree generated for the sequences in the complete genome. Phylogenetic tree constructed on the basis of the complete genome sequence. All sequences were collected from GenBank. The virus detected in this study was marked with black triangle. Trees were prepared using the Treeview programs and all branches supported based on 100 bootstrapped data sets. 


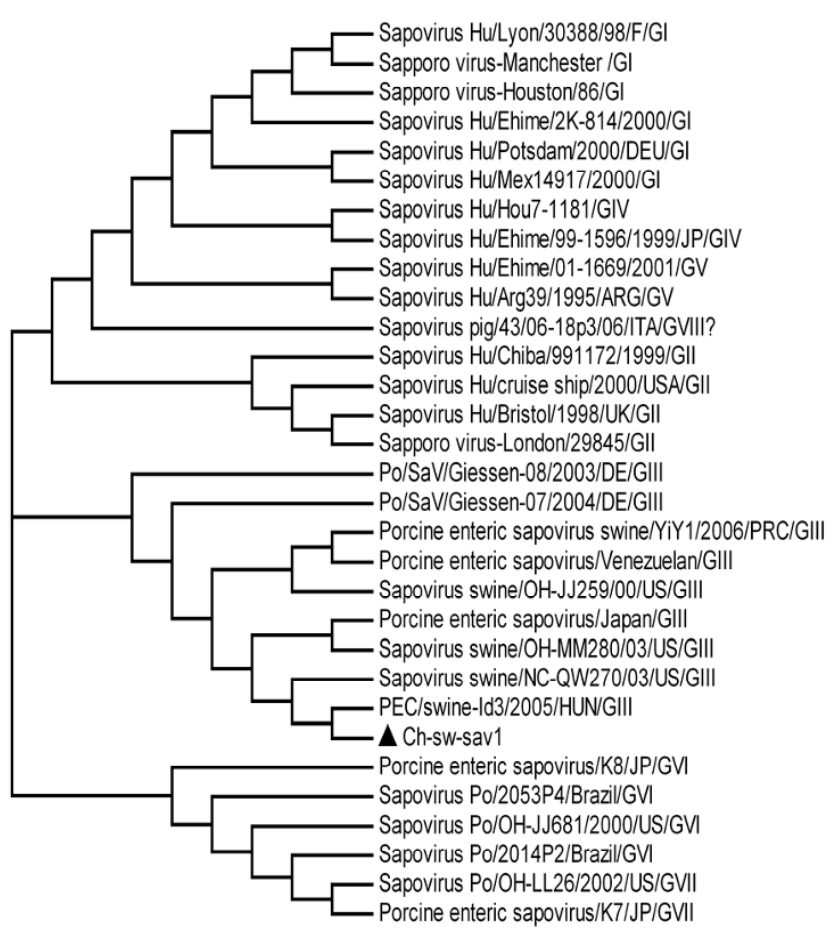

Figure 3

Unrooted phylogenetic tree of calicivirus RdRp gene sequences constructed by the neighbor-joining method. Phylogenetic tree constructed on the basis of concentrated RdRp gene sequence. Trees were prepared using the Treeview programs and are based on 100 bootstrapped data sets. All sequence used in this analysis were collected from GenBank. The virus detected in this study was marked with black triangle and it was composed of a cluster with $\mathrm{PEC} /$ swine-Id3/2005/HUN and Sapovirus swine/NCQW270/03/US, they also belong to porcine SaV genotype GIII.

\section{Sequence comparison}

We compared the entire genome sequence identities of Ch-sw-sav1 with those of other calicivirus, A phylogenetic tree based on the entire genome sequence showed that Ch-sw-sav1 was closely related to the SLVs than to the other caliciviruses (Fig. 2). The phylogenetic tree was then constructed on the basis of concentrated alignments of RNA dependent RNA polymerase gene sequence of $31 \mathrm{SaV}$ strains by the neighbour-joining method (Fig. 3). All eight genotypes were separated into corresponding lineages. Within the genotype-3 lineage, there were four distinct subgroups. The analysis indicated that Ch-sw-sav1 formed a subgroup together with two USA strains, one Japanese strain and one Hungary strain. Further analysis indicated Ch-sw-sav1 shared 82.2\%-91.2\% identities with the other GIII SaV strains, and it was closely related to the Hungary variant DQ383274 (Table 3). Whereas, it was less similar $(<57.1 \%)$ to the strains of GI, GII, GIV, GV, GVI, GVII, GVIII.

The 5 ' terminus of the genomic and predicted subgenomic RNAs of Ch-sw-sav1 possessed leader sequences with a Kozak structure (G/ANNATGG), which was favourable for translation initiation of eukaryotic mRNA [22] (Fig. 1B), similar to that of PEC (GenBank No.: AF182760) [13], The VP1 region (544aa) of Ch-sw-sav1 was the same in length as in PEC and slightly shorter than those of SaVs of human origin. The ORF2 overlapped 4 nucleotides with VP1 gene, common to others in PEC (Fig. 1C), but the length of ORF2 was distinct. Sequence alignment based on the 3' end of ORF2 of six available sequences in GenBank indicated that there was 21-nt long nucleotide sequence insertion, which was similar to the gene module of OH-JJ-259-00-US strain (GenBank No.: AY826423) with 27-nt long nucleotides inserted (Fig. 4). Analysis of antigen index showed that the inserted sequence was

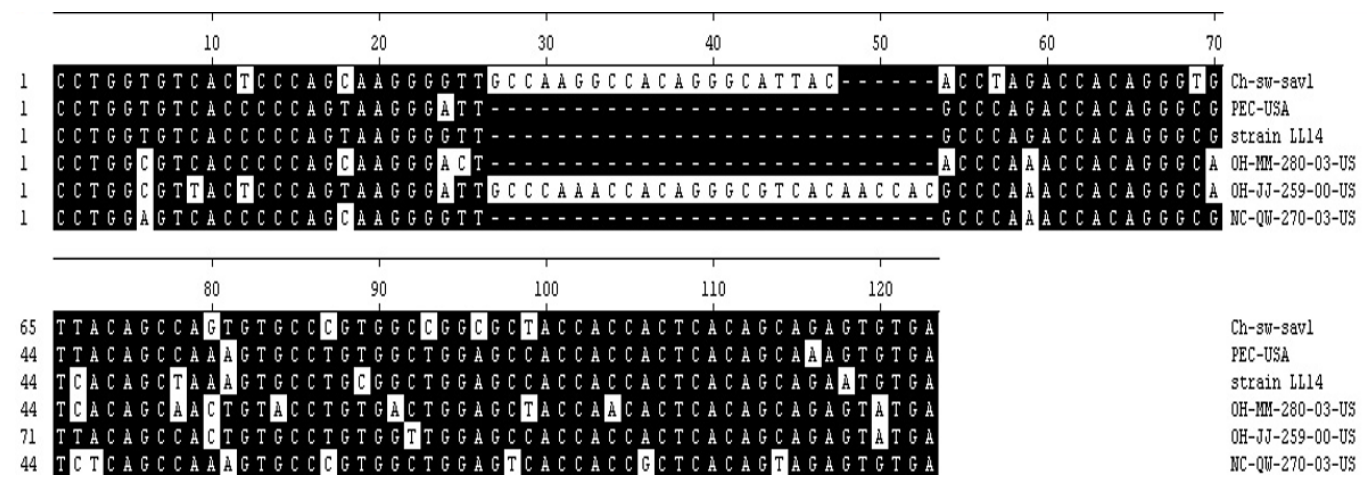

Figure 4

Nucleotide acid alignment of 3' end sequences of VP2 among six porcine SaV strains. The numbers above the alignment show the nucleotide location in the ORF2. The nucleotide with the white background is differential. The inserted sequence of Ch-sw-savl is from 27-nt to 46-nt 


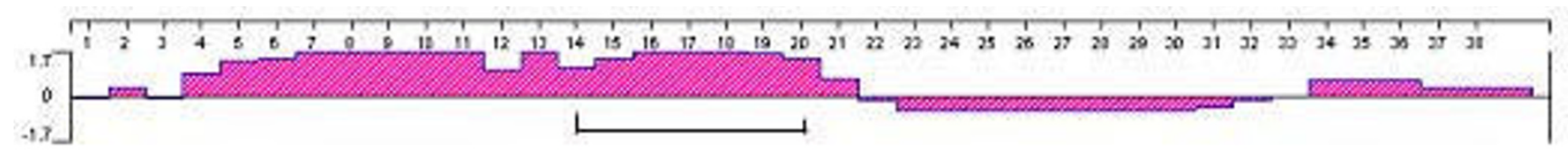

\section{Ch-sw-sav1}

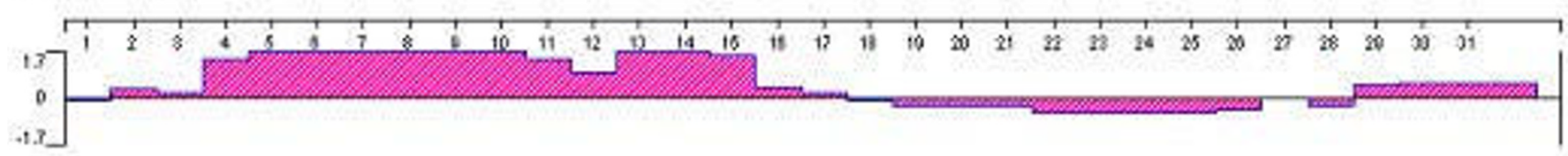

NC-QW-270-03-US

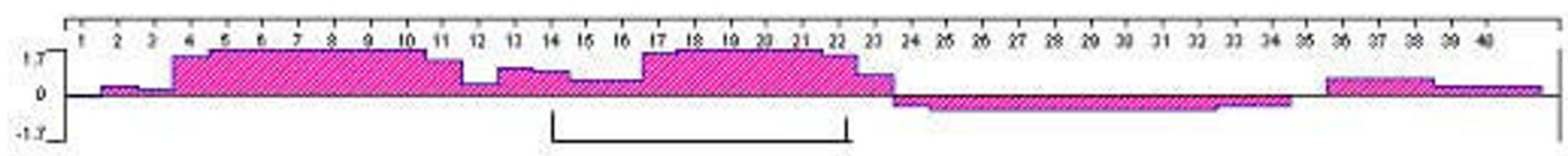

OH-JJ-259-00-US

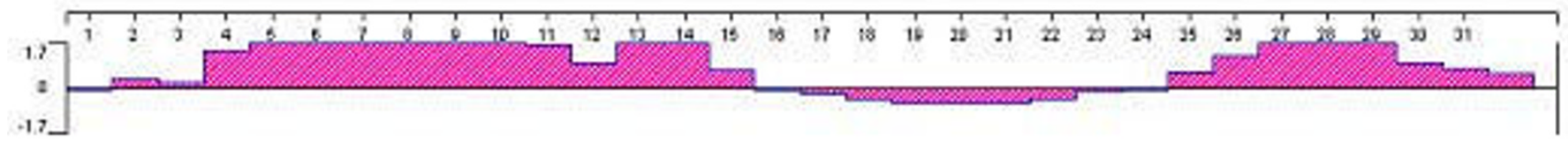

OH-MM-280-03-US

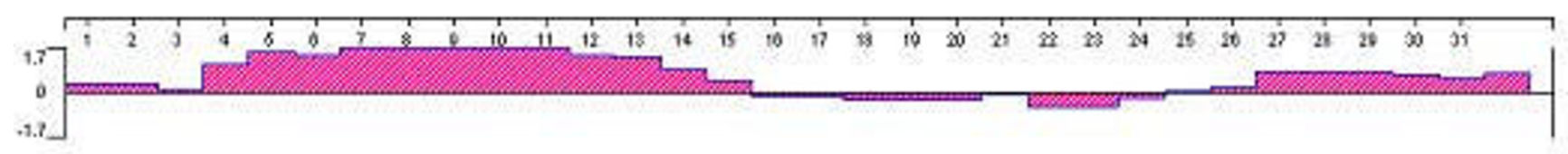

\section{PEC-USA}

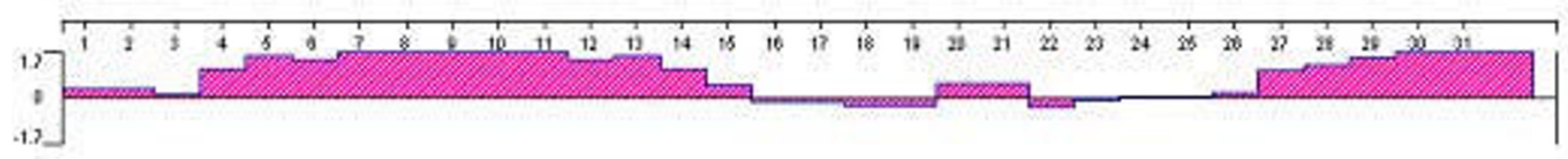

\section{strain LL14}

\section{Figure 5}

Antigen index analysis of 3' end sequences of VP2 among six porcine SaV strains. Antigen index is analysed by protean using DNAstar software. The regions marked by scale are the site of inserted sequence.

within the affluent antigen site besides another at the 3' end of ORF2 (Fig. 5).

\section{Discussion}

Sapporo virus was identified in 1982 from an outbreak of diarrhea in an orphanage in Sapporo, Japan [23]. Schuffenecker [24] classified them into three major genetic groups. Furthermore, it has been divided into eight genogroups based on the genetic diversity of the viral polymerase [25]. PEC, the first of pig origin, was discovered in 1980 s in the United States and belongs to SaV GIII [12].
Hitherto, SaV has been identified in many countries [1418]. Traditionally, we thought only SaV GIII infected pig. However, strains detected in USA and Italy that belonged to new genotype showed high homology with human SaVs respectively. It indicated that animals might act as reservoirs for human caliciviruses. So it is necessary to analyze the genetic profile of porcine $\mathrm{SaV}$ for the first step of controlling the pathogen. In February 2008, we reported the first outbreak of gastroenteritis caused by porcine $\mathrm{SaV}$ in piglets in China mainland. It may be caused by simultaneous contact with virus polluted water 
or food and the virus gene profile was further investegated. Ch-sw-sav1 was chosen to be sequenced and compared with other SaV published. Results showed that it shared high homology with PEC for the similar gene structure and similar sequence motif at 5 ' terminus that was favorable for translation initiation of eukaryotic sequence [22]. However, there was 21-nt nucleotide insertion at the 3' end of ORF2 of Ch-sw-sav1. The inserted sequence had a high antigenicity index analyzed with DNAstar software. It's predicted that ORF2 encodes capsid protein that is correlative with the assembly, antigenicity and receptor interations of $\mathrm{SaV}$. So the inserted sequence may affect antigenicity profile or other profiles of capsid protein which need to be further identified [1]. Accordingly, in phylogenetic analysis, we classified Ch-sw-sav1 into Genogroup III of SaV basing on the partial RdRp gene sequence, and it shared highest nucleotide identity with the Hungary SaV (91.2\%) which was isolated from a diarrheaed pig [17].

The porcine SaV strain in the present study came from an outbreak of gastroenteritis in piglets group, which had inserted sequence at the 3' end of ORF2. The role of the inserted sequence was unknown, but it is highly divergent in sequence and differs in size in caliciviruse s. Since the ORF2 protein is functionally conserved and may be involved in protein-protein interactions or proteinnucleic acid interactions during replication based on its strong positive charge. The inserted sequence likely has special biological function. So establishing full-length infectious clones containing or not containing this inserted fragment would now be the next step towards the identification of this fragment involved in symptomatology and pathogenicity.

\section{Conclusion}

Complete sequence of the first Chinese porcine SaV was determined and analyzed providing a gene profile of porcine $\mathrm{SaV}$ presented in swine population in China today. Sequence analysis showed that it was classified into genogroup III with two ORFs. A 21-nt insertion in ORF2 changed antigenicity index of capsid protein.

\section{Competing interests}

The authors declare that they have no competing interests.

\section{Authors' contributions}

All authors participated in the planning of the project. XH was the leader of the project. SY and WZ amplified the complete genome and analyzed the genome profile. QS and $\mathrm{FH}$ went on the sequence alignment. All authors read and approved the final manuscript.

\section{Acknowledgements}

This work was supported by Key Project of Shanghai Science and Techology Committee of China under Grant No.0639 I 9 I 2 I.

\section{References}

I. Chen R, Neill JD, Estes MK, Prasad BV: X-ray structure of a native calicivirus: structural insights into antigenic diversity and host specificity. Proc Natl Acad Sci USA 2007, I 03(2 I):8048-8053.

2. Fullerton SW, Blaschke M, Coutard B, Gebhardt ], Gorbalenya A Canard B, Tucker PA, Rohayem J: Structural and Functional Characterization of Sapovirus RNA-Dependent RNA Polymerase. J Virol 2007, 8I(4): |858-|87|.

3. Bajolet $\mathrm{O}$, Chippaux-Hyppolite $\mathrm{C}$ : Rotavirus and other viruses of diarrhea. Bull Soc Pathol Exot 1998, 9 I(5 Pt I-2):432-437.

4. Guo M, Evermann JF, Saif LJ: Detection and molecular characterization of cultivable caliciviruses from clinically normal mink and enteric caliciviruses associated with diarrhea in mink. Arch Virol 200I, I 46(3):479-493.

5. Hansman GS, Oka T, Sakon N, Takeda N: Antigenic Diversity of Human Sapoviruses. Emerg Infect Dis 2007, I 3(I 0): I5 I9- I 525.

6. Chang KO, Sosnovtsev SV, Belliot G, Kim Y, Saif LJ, Green KY: Bile acids are essential for porcine enteric calicivirus replication in association with down- regulation of signal transducer and activator of transcription. Proc Natl Acad Sci USA 2004, I 0 I (23):8733-8738.

7. Farkas T, Zhong WM, Jing $\mathrm{Y}$, Huang PW, Espinosa SM, Martinez N, Morrow AL, Ruiz-Palacios GM, Pickering LK, Jiang X: Genetic diversity among sapoviruses. Arch Virol 2004, I 49(7): I 309-I 323.

8. Parashar UD, Hummelman EG, Bresee JS, Miller MA, Glass RI: Global illness and deaths caused by rotavirus disease in children. Emerg Infect Dis 2003, 9(5):565-572.

9. Farkas T, Nakajima S, Sugieda M, Deng A, Zhong W, Jiang X: Seroprevalence of noroviruses in swine. J Clin Microbiol 2005 , 43(2):657-66I.

10. Smith AW, Iversen PL, Skilling DE, Stein DA, Bok K, Matson DO: Vesivirus viremia and seroprevalencein humans. I Med Viro 2006, 78(5):693-70I.

II. Wang Q-H, Han MG, Cheetham S, Souza M, Funk JA, Saif LJ: Porcine noroviruses related to human noroviruses. Emerg Infect Dis 2005, I I ( I 2): | 874- | 88|.

12. Saif LJ, Bohl EH, Theil KW, Cross RF, House JA: Rotavirus-like, calicivirus-like, and $23-\mathrm{nm}$ virus-like particles associated with diarrhea in young pigs. J Clin Microbiol 1980, I 2(I): I05-I I I.

13. Guo M, Chang KO, Hardy ME, Zhang Q, Parwani AV, Saif LJ: Molecular characterization of a porcine enteric calicivirus genetically related to Sapporo-like human caliciviruses. J Virol 1999, 73( I I):9625-963I.

14. Jeong C, Park SI, Park SH, Kim HH, Park SJ, Jeong JH, Choy HE, Saif LJ, Kim SK, Kang MI, Hyun BH, Cho KO: Genetic Diversity of Porcine Sapoviruses. Vet Microbiol 2007, I 22(3-4):246-257.

15. Kim HJ, Cho HS, Cho KO, Park NY: Detection and molecular characterization of porcine enteric calicivirus in Korea, genetically related to Sapovirus. I Vet Med B Infect Dis Vet Public Health 2006, 53(4): I55-I59.

16. Martinez MA, Alcala AC, Carruyo G, Botero L, Liprandi F, Ludert JE: Molecular detection of porcine enteric caliciviruses in Venezuelan farms. Vet Microbiol 2006, I I 6( I-3):77-84.

17. Reuter G, Bíró H, Szucs G: Enteric caliciviruses in domestic pigs in Hungary. Arch Virol 2007, I52(3):6||-6|4.

18. Mauroy A, Scipioni A, Mathijs E, Miry C, Ziant D, Thys C, Thiry E: Noroviruses and sapoviruses in pigs in Belgium. Arch Virol 2008, I53( I 0): | $927-31$.

19. Costantini V, Loisy F, Joens L, Le Guyader FS, Saif LJ: Human and Animal Enteric Caliciviruses in Oysters from Different Coastal Regions of the United States. Appl Environ Microbiol 2006, 72(3): $1800-1809$

20. Martella V, Lorusso E, Banyai K, Decaro N, Corrente M, Elia G, Cavalli A, Radogna A, Costantini V, Saif LJ, Lavazza A, Di Trani L, Buonavoglia C, Cavalli A, Radogna A, Costantini V, Saif LJ, Lavazza A, Di Trani L, Buonavoglia C: Identification of a Porcine Calicivirus Related Genetically to Human Sapoviruses. J Clin Microbiol 2008, 46(6): 1907-1913.

21. Zhang W, Shen Q, Hua X, Cui L, Liu J, Yang S: The first Chinese porcine sapovirus strain that contributed to an outbreak of gastroenteritis in piglets. J Virol 2008, 82( I 6):8239-8240.

22. Kozak M: Structural features in eukaryotic mRNAs that modulate the initiation of translation. I Biol Chem 22, 266(30): 19867-19870. 
23. Nakata S, Chiba S, Terashima H, Sakuma $Y$, Kogasaka R, Nakao T: Microtiter solidphase radioimmunoassay for detection of human calicivirus in stools. J Clin Microbiol 1983, I 7(2): I 98-20I.

24. Schuffenecker I, Ando T, Thouvenot D, Lina B, Aymard M: Genetic classification of "Sapporo-like viruses". Arch Virol 200I, I 46(II):2II5-2I32.

25. Martella V, Lorusso E, Banyai K, Decaro N, Corrente M, Elia G, Cavalli A, Radogna A, Costantini V, Saif LJ, Lavazza A, Di Trani L, Buonavoglia C: Identification of a Porcine Calicivirus Related Genetically to Human Sapoviruses. J Clin Microbiol 2008, 46(6): $1907-1913$.

Publish with Bio Med Central and every scientist can read your work free of charge

"BioMed Central will be the most significant development for disseminating the results of biomedical research in our lifetime. "

Sir Paul Nurse, Cancer Research UK

Your research papers will be:

- available free of charge to the entire biomedical community

- peer reviewed and published immediately upon acceptance

- cited in PubMed and archived on PubMed Central

- yours - you keep the copyright

Submit your manuscript here:

http://www.biomedcentral.com/info/publishing_adv.asp
BioMedcentral 